\title{
TRANSDISCIPLINARIEDAD Y BIOÉTICA. DOS FORMAS DE ASUMIR EL DESAFÍO PLANETARIO $^{1}$
}

\author{
TRANSDISCIPLINARITY AND BIOETHICS. TWO FORMS TO TAKE ON \\ THE PLANETARY CHALLENGE
}

\author{
SERGIO NÉSTOR OSORIO GARCÍA \\ Universidad Militar Nueva Granada \\ sergio.osorio@unimilitar.edu.co
}

\author{
RECIBIDO: 05/08/2016 \\ ACEPTADO: 01/09/2016
}

Resumen: El conocimiento transdisciplinar y la bioética global, -desde los postulados ontológicos y epistemológicos que le son propios a cada una de ellas-, tiene la pretensión de brindarnos un "dispositivo" para que los humanos de la era actual podamos cultivar aquella cualidad humana profunda, que a la fecha se encuentra subdesarrollada, y que de no cultivarse de manera explícita en lo personal y en lo colectivo, la humanidad, a largo plazo, no podrá hacerse viable desde el punto de vista axiológico. En lo que sigue haré el siguiente desarrollo: en un primer momento, y apoyándome en algunos autores provenientes de la filosofía, la sociología y la bioética, pondré como telón de fondo la amenaza que se cierne sobre la humanidad actual; en segundo lugar, haré una presentación de la propuesta de un conocimiento transdisciplinar que viene desarrollando el físico rumano Basarat Nicolescu, y que apenas empieza a ser conocida por los hispanoparlantes, y en tercer lugar, postularé a la bioética como un emergente conocimiento transdisciplinar.

Palabras clave: Conocimiento transdisciplinar, bioética, desafíos planetarios

\begin{abstract}
The transdisciplinary knowledge and global bioethics, -from the ontological and epistemological assumptions that are unique to each one of them, does claim to give us a "device" for humans of the current era we cultivate that deep human quality, which to date is underdeveloped, and not explicitly grown personally and collectively, humanity, long term, may not be feasible from the point of view axiológico. In the following will the next development: at first, and leaning in some authors from philosophy, sociology and bioethics, I'll backdrop of the threat that hangs over humanity today; secondly, I will make a presentation of the proposal of a transdisciplinary knowledge that has been developing the physical Basarat Romanian Nicolescu, and thirdly, postulate bioethics as an emerging transdisciplinary knowledge.
\end{abstract}

Keywords: Transdisciplinary knowledge, bioethics, global challenges

1 El presente texto es producto del proyecto INV-HUM 1804: RIESGO, TEMOR Y PREOCUPACIÓN. Los imperativos bioéticos de la tecno-logía en la era planetaria, avalado por la Vicerrectoría de la Investigaciones de la Universidad Militar Nueva Granada, Bogotá-Colombia. 


\section{El drama humano en la Era de la civilización tecno-científica.}

El diagnóstico realizado tanto por Corbí y Nicolescu (Osorio, 2015b, 213-252), como por Potter y Morin (Osorio, 2014b), sobre la humanidad actual, y de manera específica de las sociedades europeas contemporáneas que se hacen viables a través de la producción de conocimiento científico, es simple en su formulación: se trata de sociedades que viven de la creación constante de conocimientos $\mathrm{y}$, dadas las condiciones socio-laborales y económicas implementadas desde el modelo de sociedad neoliberal, es el conocimiento tecno-científico ${ }^{2}$ el que asociado al interés de lucro de unos cuantos en detrimento de todos, está liderando y jalonando la lógica cultural de las sociedades de conocimiento, sin ningún miramiento axiológico. Es decir, sin postular y discernir los fines y valores comunes que pueden motivar a los humanos para vivir a la manera humana.

Así las cosas, las actuales sociedades europeas,-aunque no sólo ellas-, son sociedades de conocimiento, (porque viven de la creación continua de conocimientos científico-técnicos tecno-científicos) como de los productos y servicios que se pueden generar desde aquellos como motor de la economía. Pero, al mismo tiempo, son sociedades que se encuentran desmanteladas axiológicamente (Corbí, 2012b; Nicolescu, 1996; Potter, 1971, 1988). Son sociedades que no tienen cómo direccionarse axiológicamente. Si la humanidad no recupera y cultiva esta dimensión axiológica constitutiva, no podrá hacerse viable o por lo menos no podrá hacerse viable a la manera humana. Pues, de seguir con esa lógica actual terminará autodestruyéndose.

Las sociedades de la "segunda modernización", vistas éstas desde la perspectiva del sociólogo alemán Ulrich Beck, son sociedades que a través de sus herramientas tecno-científicas tienen el potencial destructor para acabar con todo aquello que no esté en su lógica acumulativa, y con ello, están poniendo en grave peligro la sostenibilidad planetaria. Dicho de otra manera, las sociedades de la "segunda modernización", son sociedades de riesgo (Beck, 2006 a, 2006b). Son sociedades que viven de producir conocimiento direccionado única y exclusivamente al lucro y al crecimiento económico de unos cuantos en detrimento de la totalidad (Beck, 2008) ${ }^{3}$.

\footnotetext{
${ }^{2}$ Nicolescu comprende por cultura tecno-científica al matrimonio entre la ciencia fundamental y la tecnología actual desde los intereses desmedidos del capital y del lucro. Es decir, desde el imperativo tecnológico que nos dice que todo lo que se puede hacer, se hará, no importa que sea para bien o para mal de la humanidad. El todo es que se pueda hacer.

${ }^{3}$ Aunque Wikipedia no sea una fuente primaria de consulta y muchos de sus datos estén sujetos a corroboración, me parece muy sugerente la presentación que se hace de sociólogo Ulrich Beck, y esto
} 
Esta situación, aunque con un aparato metodológico muy distinto, también había sido elaborada en la década de los ochenta del siglo pasado por dos sociólogos que trabajan desde la perspectiva de la sociología fenomenológica inaugurada por Alfred Schütz. Me refiero al sociólogo vienés, residente en

por la proximidad con los planteamientos tanto de Corbí como de Nicolescu para realizar un diagnóstico de la sociedad actual. Por ello, transcribo lo que allí aparece: "Características de la 'Sociedad del Riesgo'. Definición: Fase de desarrollo de la sociedad moderna donde los riesgos sociales, políticos, económicos e industriales tienden cada vez más a escapar a las instituciones de control y protección de la sociedad industrial. Podemos distinguir ocho características destacadas: 1.Los riesgos causan daños sistemáticos a menudo irreversibles. 2. El reparto e incremento de los riesgos sigue un proceso de desigualdad social. 3. Riesgo, negocio con doble causa; riesgo y oportunidades de mercado. 4. Hay un vacío político e institucional. Los movimientos sociales son la nueva legitimación. 5. Las fuentes que daban significado colectivo a los ciudadanos están en proceso de "desencantamiento". 6. En las nuevas sociedades recae en el individuo un proceso de "individualización" a través de una desvinculación de las formas tradicionales de la sociedad industrial y una re-vinculación con otro tipo de modernización. 7. Las fuentes colectivas que dan significado a la sociedad se agotan y el individuo, busca de forma independiente, una identidad en la nueva sociedad. En situaciones de clase el ser determina la conciencia, mientras que en situaciones de riesgo es al revés, la conciencia determina el ser. 8. Retorno de la incertidumbre; riesgo como reconocimiento de lo impredecible y de las amenazas de la sociedad industrial. En la sociedad reflexiva, la sociedad se convierte en un problema para sí misma. La sociedad reflexiva se convierte en un concepto post-industrial el cual no habían pensado los clásicos como Max Weber. Su Pensamiento. Se puede ver cómo a lo largo de sus obras Beck se sitúa en una posición crítica, enfrentándose a las corrientes del postmodernismo. De esta manera, y junto con Giddens, Bauman y Mendez, defiende a la sociología reflexiva, para no abandonar el análisis crítico frente a los problemas actuales. Beck se acerca a los problemas de la nueva sociedad, que no son los mismos que describía la sociología de las sociedades precedentes, y encuentra una fuente de incertidumbre, inseguridad y riesgos. La sociedad postmoderna asume una carga de riesgo en su propia identidad que encierra una grave contradicción: el peligro de supervivencia de la especie. La "rentabilidad" del sistema corre el riesgo de la incertidumbre; no parte de la asunción de la seguridad y de un escenario sostenible en términos ecológicos, sino que conoce que existen márgenes de peligrosidad para la especie cuya cobertura no es, paradójicamente, prioritaria en un sistema guiado por la obtención de beneficios y una representación retórica de la racionalidad que oculta la racionalidad. Los medios de comunicación, tienen un papel muy importante en la representación de los riesgos y la búsqueda de soluciones, aumentando el poder y el control social. El pensamiento de Beck está marcado por las constantes de una sociedad sometida a fuertes riesgos y a procesos de individualización. Para él la actualidad se forma con las noticias de las catástrofes ecológicas, las crisis financieras, el terrorismo, las guerras preventivas. Beck distingue una primera modernización, que discurre a lo largo de la industrialización y la creación de la sociedad de masas, de una 'segunda modernización', propia de una sociedad que tiende a la globalización y está en constante desarrollo tecnológico. En la era industrial la estructura cultural y social era la familia, pero luego ese núcleo se rompe dando lugar a la individualización, aumentándose la incertidumbre del individuo en la sociedad de riesgo. Parece ser que esto es producto del neoliberalismo económico, y no solo afecta al plano personal sino que también afecta a las instituciones.”. tomado de https://es.wikipedia.org/wiki/Ulrich_Beck. Consultado 05122015 . 
Estados Unidos, Peter Berger; y al sociólogo esloveno, también residente en Estados Unidos, Thomas Luckmann ${ }^{4}$.

En el año 1973, Berger realizó un estudio muy sugerente sobre las transformaciones de la conciencia religiosa en la sociedad moderna y allí postuló la hipótesis según la cual la sociedad moderna es una sociedad anómica ${ }^{5}$ (Corbí, Nicolescu, Morin y Potter dirían que es una sociedad desmantelada axiológicamente).

Desde el punto de vista filosófico, el pensador español José Ortega y Gasset, nos había invitado a pensar la crisis de la sociedad europea como una sociedad de incertidumbre. En sus análisis, nos dice que la crisis de la sociedad europea actual, -y no sobre decirlo, aunque no sólo ella-, es que no sabe lo que le pasa, y esto es, justamente, lo que nos pasa. En efecto, los seres humanos sin el reconocimiento y cultivo de la dimensión axiológica de la existencia, no sólo carecen de la motivación necesaria para vivir, sino que además, sin esta orientación, se pueden convertir en unos superdepredadores capaces de acabar hasta con sus propias posibilidades de vida.

En estas condiciones, como decimos coloquialmente en Colombia, "si la persona (aquí la sociedad) no sabe para dónde va, entonces, cualquier Bus (medio de transporte público) le sirve". Si la humanidad, como comunidad de destino planetario, no determina dialogalmente, en las condiciones actuales, sus finalidades propias para constituirse en comunidad humana, entonces no sólo andará errante, sino que al mismo tiempo maximizará sus potencialidades de autodestrucción (Morin, 1993). No sólo de sí misma, sino del planeta en el que ella habita (Jonas, 1995; Osorio, 2014a).

La humanidad no se hará viable, humanamente hablando, única y exclusivamente con la creación de conocimiento tecno-científico, sino que ha de desarrollar otro tipo de conocimiento que le permita hacer un uso razonable del conocimiento científico (Potter, 1971; 1988). De no ser esto posible, la humanidad como un todo, desaparecerá sobre la faz de la tierra. Todo será cuestión de tiempo.

\footnotetext{
${ }^{4}$ Peter Ludwing Berger es un teólogo luterano y sociólogo que se hizo muy famoso con el libro, escrito a dos manos con Thomas Luckmann sobre la construcción social de la realidad (Berger \& Luckmann, 1967, en castellano: Berger \& Luckmann, 1986). Desde 1981 Berger es profesor de sociología y teología en la Universidad de Boston. Desde 1985 ha sido Director del Instituto para el Estudio de la Cultura Económica, que con el tiempo y ayuda de Berger cambió de nombre. Hoy se conoce como el Instituto de Cultura, Religión y Asuntos Mundiales.

${ }^{5}$ Una buena introducción al asunto planteado por Berger se puede encontrar en: Bonete Perales, Enrique (1990, pp. 557-590); y Wuthnow et al. (1988, pp. 17-19; 31-90; 267-291).
} 


\section{La crisis axiológica vista desde la racionalidad científica moderna y el surgimiento del modelo disciplinar de Universidad}

Un modo específico de organización social, de gobernabilidad y de racionalidad, fueron los determinantes fundamentales para la naciente sociedad moderna europea.

Este proceso que se fraguó en un periodo de varios siglos a través de las complejas relaciones entre la organización, consolidación y expansión de una economía de mercado capitalista; la organización de la comunidad política a través de la constitución de los estados nacionales, de regímenes políticos democráticos y la producción de un tipo de conocimiento científico, dieron origen a la creación de una de las instituciones más representativas de la modernidad: las instituciones de educación superior o dicho de manera muy simple, el modelo disciplinar de universidad.

El hombre de la modernidad convertido en sujeto racional, pretendió ser capaz de observar el mundo que tenía frente de sí, sin ningún filtro personal, y aunque esto era epistemológicamente imposible, creyó y actúo como si dicha posibilidad fuera una realidad. El conocimiento racional en el que el sujeto no se mezcla en lo conocido a la manera en que un espejo no se mete en la imagen por el reflejada o en la que un pintor no se mete en su cuadro que está pintando, es el supuesto epistemológico que se encuentran a la base de la creación de la universidad, tal y como la conocemos hoy en día.

La ciencia clásica creyó en la posibilidad de re-presentar en la mente de quien conoce una imagen que es copia fiel de lo por ella conocido, y aunque esto era epistemológicamente imposible, la reflexión filosófica de aquel entonces lo impuso como presupuesto fundamental del conocimiento racional. Nos dice Locke:

Supongamos que la mente es, como nosotros decimos, un papel en blanco, vacío de caracteres, sin ideas ¿Cómo se llena? A esto respondo con una palabra: con la experiencia. En ella se funda todo nuestro conocimiento, y de ella se deriva todo, en último término. Nuestra observación, ocupándose ya sobre objetos sensibles externos o ya sobre las operaciones internas de nuestras mentes, percibidas y reflejadas por nosotros mismos, es la que abastece a nuestro entendimiento con todos los materiales del pensar. Cuando las ideas simples se ofrecen a la mente, el entendimiento no puede rehusar tenerlas, ni alterarlas cuando están impresas, ni borrarlas para hacer otras nuevas, de la misma manera que un espejo no puede rehusar, alterar o destruir las imágenes o ideas que los objetos puestos delante de él producen (Locke $2000^{2}$ : 250). 
La imagen representacionista del conocimiento que supone, por un lado, que el conocimiento es reflejo fiel del mundo observado -sin el observador-, y que el sujeto es totalmente pasivo, -como el espejo-, para percibir y concebir la realidad exterior, (supuesto de todo el edifico del conocimiento moderno), no es capaz de abordar la experiencia humana en su totalidad, sino solo aquella que compromete la dimensión del conocimiento identificado con modelo experimental de la naturaleza (el universo) que funciona al estilo de una máquina perfectísima.

El ideal de la ciencia clásica centra su atención en las teorías objetivas (ausentes de sujeto), que pueden ser expresadas a través de lenguajes matemáticos (sistemas legaliformes) a su vez producto de verificaciones experimentales de la realidad que tienen a la mano, -como instrumental epistemológico-, el principio aristotélico de no-contradicción. Comprender el mundo con independencia de quien lo está comprendiendo es pues, el gran avance de la racionalidad científica moderna, y al mismo tiempo la dificultad que hoy tenemos que superar, si en realidad deseamos alcanzar una sostenibilidad planetaria.

En síntesis, las características básicas que permitieron la consolidación del ideal de la racionalidad de la ciencia clásica y subsiguientemente el surgimiento de la institución universitaria moderna son en esencia las siguientes (Cfr. Osorio 2013d, 165-186):

a) El primado de un sujeto racional, éste entendido como fundamento absoluto para producir un conocimiento científico en diálogo experimental y lógico con la realidad.

b) La idea de un universo perfecto capaz de ser conocido mediante fórmulas matemáticas con carácter objetivo (ausencia del sujeto), lo que a su vez supone que la naturaleza (objeto de conocimiento), es concebida como una máquina perfectísima que se puede conocer a través de leyes invariables y permanentes y que dan la solidez al conocimiento. La naturaleza (para la modernidad el universo), es semejante a sí mismo en todas sus manifestaciones y por tanto, puede ser explicado a partir de leyes simples y recurrentes, que durante un tiempo bastante prolongado, se identificaron con las formuladas por la física mecánica. Por ello, la comprensión racional del mundo, -claramente alcanzada en la ciencia física-, legitimó el saber científico como único saber válido, en detrimento, exclusión y negación de otros saberes que se daban en la vida cotidiana, pero que no contaban para su expresión de lenguajes matematizados.

c) La separación tajante entre el conocimiento científico y el conocimiento del sentido común. Todo lo anterior, condujo a la separación irreconciliable entre el conocimiento científico, (propio de los hombres de ciencia) y el conocimiento del común, (propio del ciudadano de a pie). 
La universidad moderna a través de sus Facultades y de sus programas disciplinares, separados unos de otros, será la encargada, mediante la trasmisión y enseñanza de saberes disciplinares, de mantener la división entre la cultura de expertos y de la vida cotidiana, relegando a los ciudadanos de a pie a la condición de simples receptores pasivos de conocimiento y de sus avances técnicos-científicos.

d) La racionalidad moderna estableció la disyunción sujeto/objeto como principio ontológico y epistemológico para el conocimiento humano e implantó la evidencia lógica y el lenguaje matematizado como criterio para el conocimiento objetivo del universo.

e) Y finalmente, la racionalidad moderna sostuvo la creencia según la cual, el saber científico era el único capaz de proveer al hombre de poder inigualable para dominar la naturaleza. Según Bacón, saber es poder y el hombre de conocimientos (el científico) ha de torturar a la naturaleza para que ella le dé sus secretos.

\section{La ruptura con la racionalidad clásica y la aparición una nueva consciencia ciudadana}

En la segunda mitad del siglo XX el surgimiento de nuevos problemas, en especial el deterioro del medio ambiente, las consecuencias negativas de la implementación de imperativo tecnológico, como el reconocimiento del uso de los conocimientos científico-técnicos con fines militares en un mundo bipolar, entre otros, fueron produciendo el surgimiento de una nueva consciencia ciudadana tanto en el científico como en el hombre de a pie, que ha cobrado vida en movimientos y grupos de presión, los que a su vez han tenido una influencia notable en a la superación de la racionalidad clásica, porque pone a la sociedad en peligro y riesgo de desaparecer (Beck, 2006a).

Estas reacciones ciudadanas plantean serios desafíos para la producción de los sistemas científicos, para la legitimidad de las instituciones sociales y para la organización de los sistemas educativos en todos sus niveles, especialmente en el nivel de la educación superior. Al mismo tiempo, implican el surgimiento de nuevas perspectivas que replantean la relación ontológica de la relación hombrehombre y hombre-naturaleza y los fundamentos epistemológicos y metodológicos para dicha relación. 
Esto significa que la organización actual del sistema de conocimientos tecnocientífico conlleva el surgimiento de problemas fundamentales que afectan la sostenibilidad de la vida humana y no-humana en el globo y que han de ser cuestionados por todos los ciudadanos del planeta. Puesto que la racionalidad científica no es una práctica neutral en relación a la vida, los impactos epistemológicos, ambientales y sociales de la creación de conocimientos científicos adquieren una nueva significación para la sostenibilidad planetaria.

Se vuelve inevitable pensar críticamente las implicaciones sociales, políticas, históricas y filosóficas de la actividad científica y de su organización institucional. Esto es lo que quieren hacer tanto el conocimiento transdisciplinar como la bioética global (Sotolongo \& Delgado, 2006).

\section{Hacia un nuevo horizonte ontológico y epistemológico para pensar la realidad. La transdisciplinariedad como modelo}

\section{La emergencia de una nueva ontología.}

Hoy partimos de un nuevo marco teórico para comprender la realidad. Los problemas desafiantes que nos presenta el mundo actual no vienen organizados en bloques disciplinarios, y además desbordan cada vez más los métodos y las teorías que se han elaborado dentro de la organización disciplinaria del saber, que a su vez justificaban una perspectiva del conocimiento simplificador, disyuntor/reductor de la complejidad de lo real.

Si la organización disciplinaria del saber nos obligaba a centrar el conocimiento del mundo desde "objetos" de conocimiento, la nueva organización nos obliga a pensar de otra manera dicha organización y a concebir la realidad de una manera totalmente distinta. Desde la segunda mitad del siglo $\mathrm{XX}$, hemos comenzado a concebir el universo ya no como un sistema perfectísimo y legaliforme, sino como un conjunto de sistemas dinámicos, donde las interacciones entre los constituyentes de los sistemas y su entorno resultan tan importantes como el análisis de sus componentes.

El universo (y ya no sólo la naturaleza) ha comenzado a dejar de ser un conjunto de "objetos" que reposan frente a un "sujeto" para convertirse en un enjambre de conexiones, interacciones, redes, emergencias, devenires, incertidumbres, contradicciones.

Ante estos desafíos, han comenzado a aparecer un nuevo campo de estudios e investigaciones vinculadas al problema de la racionalidad y de la sostenibilidad planetaria, y como consecuencia de este proceso ha ido emergiendo un nuevo y 
sugerente modo de comprender la creación y la organización del conocimiento humano: la transdisciplinariedad.

\section{La emergencia de una nueva epistemología}

Este campo, es una constelación en donde conviven de manera complementaria y contradictoria diversas teorías y propuestas metodológicas fundamentadas en diferentes marcos lógicos y epistemológicos, enraizadas a su vez en supuestos ontológicos diversos y muchas veces antagónicos. Pero, en todos estos intentos podemos encontrar como hilo conductor la búsqueda de una nueva racionalidad que supere el paradigma simplificador de la ciencia clásica y que nos permitan una salida racional ante la crisis mundial.

Ante esta nueva comprensión del universo y de la racionalidad que lo comprende, deberá de manera necesaria, darse una renovación sin precedentes en las instituciones de educación superior que viven, hoy por hoy, de los conocimientos producidos en el siglo XIX, cuando la racionalidad se tenía que construir desde la organización disciplinar de los conocimientos, -necesaria para su momento-, pero desde luego anacrónica para el nuestro.

No se trata simplemente de agrupar el aporte de un cierto número de disciplinas, como se hace frecuentemente, para generar un conocimiento multi, pluri o polidisciplinar; tampoco es suficiente, avanzar hacia un enfoque interdisciplinar que partiendo de varias disciplinas y operando de manera conjunta intercambian sus referentes metodológicos con miras a la resolución de un problema especial de conocimiento científico.

Se requiere de algo más: de una visión y de una metodología transdisciplinar del conocimiento que centre sus esfuerzos en la comprensión compleja del mundo y en donde una de sus condiciones de posibilidad ha de ser la integración epistemológica de los conocimientos dispersos en disciplinas. Hoy tenemos que avanzar hacia una construcción transdisciplinar del conocimiento que nos permita una nueva comprensión de la realidad que somos y nos constituye, y que nos posibilite una nueva manera de habitar la tierra en esta nuestra era planetaria.

Vistas las cosas desde el ámbito universitario, nos encontramos en medio de una gran paradoja consistente en que la universidad actualmente sigue enseñando la apropiación disciplinaria de los saberes desde la organización administrativa de Facultades, programas y departamentos, sin vínculo alguno entre ellos, mientras que la realidad que tenemos que pensar hoy se nos ha vuelto transdisciplinar, multireferencial e indisciplinada.

Sin embargo, ante esta paradójica situación ha de quedar claro que la solución no ha de ser dejar de producir conocimientos, sino producir más 
conocimientos, pero no conocimientos disciplinares, sino transdisciplinares; tampoco se trata de desechar los conocimientos disciplinares como si fueran algo repugnante, sino de buscar y encontrar una nueva forma de organizarlos.

La disciplinariedad es la organización del conocimiento científico a partir de campos o áreas especializadas del saber. Las disciplinas operan el conocimiento de las partes de un todo que por su inmensidad se hace imposible de comprender como todo. Descartes con sus reglas para guiar el espíritu hacia un saber verdadero e indubitable ha sido el mejor exponente de este tipo de mentalidad.

Las universidades modernas, son la institucionalización necesaria para la transmisión y enseñanza de los conocimientos alcanzados de manera disciplinar o mejor aún, son las instituciones sociales que transmiten de manera pedagógica la adquisición de un saber construido y organizado de manera disciplinar. Para ello, administrativamente crearon las unidades académicas llamadas Facultades, Departamentos, Institutos o Escuelas, que pedagógicamente enseñan y trasmiten una serie de saberes fragmentados unos de otros, que se desarrollan aisladamente mediante los métodos más adecuados para el conocimiento de unos "objetos" funcionales del conocimiento.

$\mathrm{Y}$ esto, si tenemos en cuenta que el "todo" es concebido como un gran mecano, como un reloj perfectísimo en donde cada pieza funciona de manera repetitiva y sin fisuras, la comprensión no podría ser de otra manera. La ciencias clásicas y la universidad moderna, son desde esta perspectiva, las "herramientas" más eficaces y más expeditas para la producción y la enseñanza del conocimiento de un "todo" que funciona al estilo mecánico.

Pero, ¿Qué sucedería si el universo, como lo hemos visto, ya no es considerado como un mecano, sino como un polisistema que se produce y autoreproduce a partir de, desde y por medio la interacción de sus subsistemas, que se conciben a su vez por las relaciones entre ellos y por las relaciones entre ellos con un todo emergente? Sencillamente que el conocimiento disciplinar de la realidad como conocimiento de las partes y como suma de las mismas se torna profundamente inadecuado. Insistir en la construcción y enseñanza de conocimientos disciplinares, hoy por hoy, además de inadecuado, es ineficaz. La mirada profunda de las disciplinas es importante y necesaria para un cierto conocimiento de la realidad, pero insuficiente de cara la comprensión de la realidad que ha dejado de ser mecánica y que ya no puede ser comprendida de manera disciplinar.

La multi-pluri o polidisciplinariedad, también ha intentado salirle al paso a este impasse, pero al no cuestionar el presupuesto ontológico y epistemológico de la ciencia clásica, no ha logrado avanzar lo suficiente. 
La multi-pluri o polidisciplinariedad, ha buscado ir más allá del conocimiento disciplinar, pero en la práctica sólo ha podido solapar varios conocimientos en un marco más amplio al del conocimiento disciplinar.

El enfoque interdisciplinar del conocimiento ha querido ir más lejos que el enfoque polidisciplinar, pero tampoco ha podido deshacerse del carácter simplificador del paradigma disciplinar.

La construcción interdisciplinar del conocimiento, amplía el objeto de estudio desde el intercambio conceptual y metodológico de las diferentes disciplinas que acuden ante un determinado objeto de conocimiento, pero no logran ir más allá del objeto puesto que se refieren a un solo nivel de realidad o de manera excepcional a fragmentos de un único nivel de la realidad. Por tanto, el conocimiento interdisciplinar tampoco logra superar el marco de interpretación del paradigma simplificador de la ciencia clásica.

La transdisciplinariedad, hunde sus raíces en la poli y la interdisciplinariedad, pero va más allá, posibilitando una nueva articulación epistemológica del conocimiento y respondiendo a una nueva comprensión ontológica de la realidad.

La transdisciplinariedad, consiste como el prefijo "trans" indica, a lo que está a la vez entre, a través y más allá de toda disciplina. Su objetivo o finalidad es la comprensión del mundo actual, donde uno de sus imperativos es la unidad del conocimiento (Nicolescu: 1996: 38).

En último término lo que distingue y relaciona a un mismo tiempo el conocimiento transdisciplinar de los conocimientos poli e interdisciplinares es, Strictu Sensu, su finalidad: mientras estos no van más allá de su pretensión disciplinar, aunque la amplíen, el conocimiento transdisciplinar se interesa en la dinámica de la acción de varios niveles de realidad de cara la comprensión del mundo presente, que desde luego, es imposible conocer desde el paradigma disciplinar.

La transdisciplinariedad emerge cuando han sido puestos entre paréntesis las barreras demarcatorias de las disciplinas y cuando la realidad ya no es vista como un "objeto perfectísimo" que está al frente de un "sujeto conocedor" que pretende conocer de manera mecánicamente determinista.

La transdisciplinariedad es la forma de conocer la complejidad de lo real de la cual somos y hacemos parte; es la mejor estrategia cognoscitiva para poner en circulación el pensamiento capaz de complejizar la concepción disciplinar del mundo y de reintroducir al sujeto que conoce en todo proceso de conocimiento. 
El conocimiento transdisciplinar centra su atención en la comprensión multidimensional, multireferencial y polinivelada de la realidad que hace posible un "Conocimiento del conocimiento" que es a su vez la clave de bóveda de la epistemología de la complejidad y de una bioética que opera como conocimiento transdisciplinar (Osorio 2015a, 213-252).

El conocimiento transdisciplinar levanta su vuelo en las postrimerías del siglo XXI, para ayudarnos a buscar la unidad del conocimiento fragmentado en disciplinas y para afrontar desde una nueva racionalidad el reto de la supervivencia planetaria.

La transdisciplinariedad será el reto de la universidad para el siglo XXI que ahora comenzamos y del que la Universidad, desde su consciencia estratégica, tendrá que aprehender desde un nuevo sistema epistemológico de interpretación (Osorio 2013d, 165-183).

\section{La Bioética como transdisciplina.}

Con certeza, para la mayoría de nuestros lectores, la palabra bioética es una palabra muy rara. Pero, habría que decir que no sólo para nuestros lectores, sino para el público en general. La bioética es un neologismo que requiere de quien lo escucha de un esfuerzo mental para la comprensión de su significado. Por este motivo, y para un público no-especializado voy a intentar dar algunos criterios para la dilucidación del término.

La bioética es una palabra inventada por el bioquímico norteamericano Van Rensselaer Potter hacia la década de los $70^{\prime}$. Potter, en un sentido cronológico, es considerado el padre de la bioética. ¿Qué quería significar Potter con esta palabra? Con temor a ser demasiado simplificador puedo decir que Potter quería plantear mediante el término bioética la respuesta a una pregunta: ¿Hacia dónde nos llevan, como individuos y como especie, los desarrollos de la racionalidad científicotécnica? Y si acaso ¿ $¿$ Tendremos otra opción?

Ante esta pregunta Potter responderá en el año 1962, que el desarrollo científico nos llevará de manera inevitable, hacia el colapsamiento de la humanidad como especie (Potter, 1992). Los adelantos científico-técnicos en sí mismos no son malos, ni inconvenientes, por el contrario, son necesarios y muy útiles. Pero, los adelantos científicos-técnicos jalonados y supeditados a la acumulación inmediata y desmedida del capital económico de unas pocas personas y de unas pocas organizaciones, en detrimento de la humanidad, pondrán a futuro, y no tan a futuro, en serio peligro la sostenibilidad de la vida del planeta y de la vida humana como parte de la vida del planeta. Por esta razón 
La bioética debería llegar a ser un nuevo tipo de ciencia de la vida, esencialmente interdisciplinar y preocupada por la sobrevivencia de la especie humana, capaz de integrar la biología humana (ampliamente entendida), la competencia humana en crear y acompañar los valores humanos, los problemas del medio ambiente y de los otros seres vivos que intervienen en la preservación y mejoría de la propia calidad de vida de los seres humanos (Potter, 1971, 11).

En relación a la segunda pregunta: Y acaso ¿Tendremos otra opción? Potter a pesar de todo pronóstico negativo, nos dirá que la supervivencia del planeta como de la humanidad en tanto que especie, dependerá de lo que nosotros mismos podamos hacer con los adelantos científico-técnicos. Para ello se requiere que la racionalidad científico-técnica pueda ser dirigida por otro tipo de racionalidad, diríamos hoy por una racionalidad práctico-moral que determine la orientación ética de la racionalidad tecno-científica. Pero aquí justamente está el problema. Potter como científico dedicado por más de 30 años a la investigación con células cancerígenas, sabe que la humanidad se ha superdesarrollado desde el punto de vista de la racionalidad científico-técnica, pero también sabe que ha quedado subdesarrollada desde el punto de vista de la racionalidad práctico-moral. Por ello, piensa que la bioética será una nueva reflexión que superará el déficit de la racionalidad moral de la sociedad accidental

En este sentido la bioética será el nombre para una nueva sabiduría capaz de dirigir razonablemente el conocimiento científico-técnico de cara a la posibilidad de vida humana y no-humana al interior de nuestro planeta. La bioética será una nueva sabiduría que proporcione al hombre el conocimiento de cómo usar el conocimiento para la supervivencia planetaria y para la mejora de la humanidad.

La bioética deberá ser vista como un enfoque cibernético de la búsqueda continua de sabiduría, lo que yo he definido como el conocimiento de cómo usar el conocimiento para la supervivencia humana y para mejorar la condición humana... En conclusión les pido que piensen la bioética como una nueva ética científica que combina la humildad, la responsabilidad y la competencia, que es interdisciplinaria e intercultural y que intensifica el sentido de la humanidad (Potter, 1988, 32).

Pero con ello no acaban las pretensiones potterianas. Potter también sabe que la humanidad se realiza humanamente en medio de entornos o contextos socioculturales a partir de los cuales los humanos determinan aquellas cosas que no se han determinado genéticamente. El ser humano es el único animal que para poder hacerse viable tiene que determinar socio-culturalmente los cómos de su 
existencia. Por tanto, con un mínimo de base biológica, tiene que determinar culturalmente lo que le hace falta para sobrevivir. Tiene que determinar entre otras cosas, su vida sexual, su organización familiar, sus modos de relación y de organización social, económica y política, su inmersión en la totalidad del universo.

En una palabra, debe organizar los fines por los que ha de vivir: su axiología. Y ésta decididamente no se la puede dar la racionalidad científico-técnica. Es necesario acudir a otro tipo de racionalidad en la que las instancias de mediación cultural cumplan una función programadora de la vida de los humanos.

Por eso el medio cultural, construcción específica de la especie humana, deberá balancear adecuadamente el instinto individual de corto plazo y las necesidades a largo plazo de la especie para una supervivencia decente, en contraste con la mera supervivencia de una sobrevida miserable en el planeta (Potter, 1975, 54).

En este contexto cultural, como nos dirá el pensador Martin Heidegger, la racionalidad científico-técnica hace, y ese es su gran mérito. "La ciencia hace, pero no piensa"

El fundamento de este estado de cosas está en que la ciencia no piensa. No piensa porque, según el modo de su proceder y de los medios de los que se vale, no puede pensar nunca; pensar, según el modo de los pensadores. El hecho de que la ciencia no pueda pensar no es una carencia sino una ventaja. Esta ventaja le asegura a la ciencia la posibilidad de introducirse en cada zona de objetos según el modo de la investigación y de instalarse en aquélla. La ciencia no piensa (Heidegger, 1994, 117).

La racionalidad técnica no puede determinar los fines últimos de su acción. Esta es la función de la racionalidad práctico-moral. Por ello para Potter sólo la integración de la perspectiva científico-tecnológica con la perspectiva prácticomoral posibilitará en los seres humanos no sólo la viabilidad como especie, sino también su modo humano de sobrevivencia. La bioética guiará a la humanidad a lo largo de este puente hacia el futuro (Potter, 1971).

En medio del actual proceso histórico de planetarización, las cuestiones fundamentales de la humanidad: ¿Quiénes somos? ¿De dónde venimos? y ¿Para dónde vamos? se hacen nuevamente presentes y adquieren una nueva determinación no sólo para la especie humana, sino para la sostenibilidad de la vida del planeta de la que depende la vida de la especie humana.

En síntesis, la bioética ha de ayudarnos a pensar una nueva racionalidad científico-técnica como un nuevo modo socio-cultural de vida para hacernos 
viables y para hacernos humanos. Hoy la humanidad no sólo es más consciente de sus deberes y derechos para consigo misma y para con los demás, sino que concomitantemente ha tomado consciencia de que los modos de socio-culturales de vida religados a los ecosistemas de los que hacen parte, alcanzan un grado tal de auto-organización que les permite reflexivamente preguntarse sobre las posibilidades que tienen para su viabilidad y para lograr una sostenibilidad en tanto que comunidad de destino planetario.

En estas nuevas coordenadas, la bioética será no sólo la posibilitación para el cultivo de la dimensión axiológica de la existencia, (la actualización de la autonomía moral de los seres humanos), sino también y a un mismo tiempo, la animadora de la construcción de un nuevo modo humano de ser en el que sea posible una vida digna y la paz para todos los habitantes de la tierra.

Los énfasis de la reflexión bioética estarán determinados por las condiciones geo-políticas y geo-estratégicas con las que cuentan los modos socio-culturales de vida en el escenario mundial. La reflexión bioética se preguntará muy críticamente por el destino de la humanidad en medio de los mecanismos atroces de globalización neoliberal. La bioética en este sentido, será una reflexión crítica $\mathrm{y}$ al mismo tiempo un movimiento social que desenmascare los mecanismos socio-culturales que esclavizan, oprimen y llevan a la muerte a los menos aventajados de la sociedad.

\section{Bibliografía}

Beck, Ulrich (2006a). La sociedad del riesgo, Barcelona: Editorial Paidós. Beck, Ulrich. (2006b). La sociedad del riesgo: hacia una nueva modernidad, Madrid: Ediciones Paidós.

Beck, Ulrich. (2008). La sociedad del riesgo global. Madrid: Siglo XXI Editores.

Berger, P., Berger, Brigitte \& Kellner, Hansfried. (1979). Un mundo sin hogar. Modernización y conciencia. Santander: Sal Terrae.

Berger, P \& Luckmann. (1998). La construcción social de la realidad, traducción de Silvia Zuleta, Madrid: Amorrortu editores.

Berger, P \& kellner, H. (1973). The homeless mind: modernization and consciousness. New York: Randonm House.

Bonete Perales, E. (1990). "Peter L. Berger: una teoría sociológica de la modernidad". Revista religión y cultura, Barcelona, no. 175, p. 557-590.

Corbí, Marià. (2014). La necesidad ineludible del cultivo de la cualidad humana y de la cualidad humana profunda en nuestras sociedades, como una indagación 
libre en comunicación y en servicio. Justificación de la temática para el 10 encuentro internacional anual de investigadores de la red de investigación en epistemología axiológica. Documento de trabajo, 2014. Disponible en:

http://www.cetr.net/es/nuestros_libros/10_la_necesidad_ineludible_del_culti>.

Corbí, Marià. (2010b) el cultivo de la cualidad humana y de la cualidad humana profunda, boletín CETR, agosto-septiembre, Tomado de http://www.cetr.net/modules.php?name=news \&file $=$ article $\&$ sid $=657$

Corbí, Marià. (2014). Hacia una espiritualidad laica. Sin creencias, sin religiones, sin dioses, Barcelona: Herder.

Heidegger, Martin, Conferencias y artículos, Traducción de Eustaquio Barjau, Barcelona: Ediciones del Serbal, 1994, pp.113-125.

Morin, Edgar \& Kern, Anne Brigitte (1993). Tierra patria, Barcelona: Editorial Kairós.

Nicolescu, Basarat, (2014). The need for transdisciplinarity in higher education. keynote speaker talk at the international higher education congress "new trends and issues". may, 27th-29th, 2011a. istanbul, turkey. Disponible en: http://basarab.nicolescu.perso.sfr.fr/basarab/docs_articles/istanbul_uik2011.pdf Jonas, Hans (1995). El principio de responsabilidad. Ensayo de una ética para la civilización tecnológica. Barcelona: Editorial Herder.

Locke, John. $\left(2002^{2}\right)$. Tratado sobre el entendimiento humano, México: Fondo de Cultura Económica.

Morin, Edgar. $\left(1988^{2}\right)$. El Método III: el conocimiento del conocimiento, Madrid: Editorial Cátedra.

Nicolescu, Basarab (1996), La transdisciplinariedad. Manifiesto, Mónaco: Mónaco: Editions du Rocher.

Nicolescu, Basarat, (2008a). spiritual dimension of democracy. utopia or necessity? in: Marga, Andrei; Bercheim, Theodor; Sadlak, Jan (ed.). Living in truth: a conceptual framework for a wisdom society and the european construction. cluj-napoca: cluj university press, pp. 509-516.

Nicolescu, Basarat, (2007). La transdisciplinariedad como marco metodológico para ir más allá del debate entre ciencia y religión. Disponible en: <http://www.metanexus.net/essay/transdisciplinarity-methodologicalframework-going-beyond-science-religion-debate>.

Nicolescu, Basarat, (2014). La transdisciplinariedad. Manifiesto. Mónaco: Editions du Rocher. Tomado de <http://basarab.nicolescu.perso.sfr.fr/ciret/espagnol/visiones.htm>.

Osorio García, Sergio Néstor. (2013d). De las reformas pragmáticas a la transformación paradigmática de la educación superior. La transdisciplinariedad como atractor. En: Jorge Najjar-Lorena Vitola-Fabio Alberto Garzón Díaz, 
Desafíos e perspectivas na Educacao para Brasil e Colombia. Niteroi, Brasil: Intertexto Editora y consultoría, pp. 165-186.

Osorio García, Sergio Néstor. (2014a). La bioética a la luz de las epistemologías de segundo orden I: El aporte crítico de Iván Illich y Hans Jonas, Bogotá: UMNG-AF\&M Producción Gráfica S.A.S. 209 p.

Osorio García, Sergio Néstor. (2014b). La bioética a la luz de las epistemologías de segundo orden II: El aporte crítico de Edgar Morin, Marià Corbí y Carlos Castaneda, Bogotá: UMNG-Digiprint S.A.S. 107 p.

Osorio García, Sergio Néstor, "Epistemología axiológica y conocimiento transdisciplinar. Estrategias cognoscitivas para el reconocimiento y cultivo de la cualidad humana profunda y la dimensión sagrada de la existencia" Revista Horizonte, Belo Horizonte, vol. 13, no. 37, p. 213-252, Enero/Mazo 2015.

Potter, Vans Rensselaer (1988). Global bioethics: building on the Leopold Legacy. East Lansing: Michigan State University Press.

Potter, Van Rensselaer, (1975), "Humilty with responsability, a bioethics for oncologits presidential addres", Cancer Research 35.

Sotolongo Codina, Pedro \& Delgado Díaz, Carlos, (2006), La revolución contemporánea del saber y la complejidad social, Colección Campus Virtual de CLACSO, Buenos Aires, Argentina.

Wuthnow, R.; Hunter, j. d.; Bergesen, A.; Kurzweil, E. (1998). Análisis cultural: la obra de Peter L. Perger, Mary Douglas, Michael Foucault y Jürgen Habermas, Barcelona: Editorial Paidós, 1998. p. 17-19; 31-90; 267-291. 
